PAPER REFERENCE: E-MRS-spring, Oral Talk G-5 3

\title{
Influence of complex impurity centres on radiation damage in wide-gap metal oxides
}

\author{
A. Lushchik ${ }^{\mathrm{a} * 1}$, Ch. Lushchik ${ }^{\mathrm{a}}$, A.I. Popov ${ }^{\mathrm{b}}$, K. Schwartz ${ }^{\mathrm{c}}$, E. Shablonin ${ }^{\mathrm{a}}$, E. Vasil'chenko ${ }^{\mathrm{a}}$, \\ anstitute of Physics, University of Tartu, Ravila 14c, 50411 Tartu, Estonia \\ ${ }^{b}$ Institute of Solid State Physics, University of Latvia, Kengaraga 8, Riga LV-1063, Latvia \\ ${ }^{c}$ GSI Helmholtzzentrum für Schwerionenforschung (GSI), Planckstr. 1, 64291 Darmstadt,
} Germany

\begin{abstract}
Different mechanisms of radiation damage of wide-gap metal oxides as well as a dual influence of impurity ions on the efficiency of radiation damage have been considered on the example of binary ionic $\mathrm{MgO}$ and complex ionic-covalent $\mathrm{Lu}_{3} \mathrm{Al}_{5} \mathrm{O}_{12}$ single crystals. Particular emphasis has been placed on irradiation with $\sim 2 \mathrm{GeV}$ heavy ions $\left({ }^{197} \mathrm{Au},{ }^{209} \mathrm{Bi},{ }^{238} \mathrm{U}\right.$, fluence of $10^{12}$ ions $/ \mathrm{cm}^{2}$ ) providing extremely high density of electronic excitations within ion tracks. Besides knock-out mechanism for Frenkel pair formation, the additional mechanism through the collapse of mobile discrete breathers at certain lattice places (e.g., complex impurity centres) leads to the creation of complex defects that involve a large number of host atoms. The experimental manifestations of the radiation creation of intrinsic and impurity antisite defects $\left(\left.\mathrm{Lu}\right|_{\mathrm{Al}}\right.$ or $\left.\mathrm{Ce}\right|_{\mathrm{Al}}$ - a heavy ion in a wrong cation site) have been detected in LuAG and LuAG:Ce ${ }^{3+}$ single crystals. Light doping of LuAG causes a small enhancement of radiation resistance, while pair impurity centres (for instance, $\left.\mathrm{Ce}\right|_{\mathrm{Lu}}-\left.\mathrm{Ce}\right|_{\mathrm{Al}}$ or $\mathrm{Cr}^{3+}-\mathrm{Cr}^{3+}$ in $\mathrm{MgO}$ ) are formed with a rise of impurity concentration. These complex impurity centres as well as radiation-induced intrinsic antisite defects ( $\left.\mathrm{Lu}\right|_{\mathrm{Al}}$ strongly interacting with $\mathrm{Lu}$ in a regular site) tentatively serve as the places for breathers collapse, thus decreasing the material resistance against dense irradiation.
\end{abstract}

Keywords: radiation defects, non-impact mechanisms, luminescence, impurity centres, metal oxides

PACS: 61.80.Jh; 61.80.-x; 78.20.Ci; 78.60-Kn

${ }^{1}$ Corresponding Author: Tel. +372-7374619; Fax. +372-7383033;

Email: aleksandr.lushchik@ut.ee 


\section{Introduction}

The variety of radiation effects have been studied for a long time in pure and doped wide-gap materials (WGMs, gap of $E_{\mathrm{g}}=5-15 \mathrm{eV}$ ) with special emphasis on model alkali halide crystals and binary metal oxides (see, e.g., [1-9] and references therein).

The energy absorbed by the material during irradiation is only partly spent on the excitation of different intrinsic and impurity emissions. The latter are used for applications in various time scales: in scintillation detectors and spectral transformers a fast luminescence response is needed, while personal dosimetry operates with luminescence light sum released at a subsequent heating of a preliminarily irradiated WGM. A significant part of the energy gained during irradiation is transformed via nonradiative transitions into heat (package of phonons) causing complex processes of electron-phonon relaxation, local heating up to partial material melting and even evaporation, etc. One more nonradiative channel of energy dissipation is connected with the transformation of the existing structural defects or the creation of novel short-lived or long-lived (stable) lattice defects. The ratio between three above-mentioned transformation channels depends on many factors, such as chemical nature and crystalline structure, temperature, concentration and spatial distribution of luminescence centres, fundamental features of electronic excitations (EEs) in different types of materials, the density of radiation-induced EEs, etc.

The present study is devoted to a conceptual overview of our recent investigations of radiation damage induced by photons, electrons, fast neutrons and, especially, swift heavy ions (SHIs) in single crystals of binary $(\mathrm{MgO})$ and complex metal oxides $\left(\mathrm{Lu}_{3} \mathrm{Al}_{5} \mathrm{O}_{12}, 160\right.$ atoms per unit cell). Particular emphasis is laid on the features of defect creation mechanisms in SHI-irradiated ionic-covalent metal oxides with complex lattice structure as well as on a dual influence of certain impurity ions on the efficiency of radiation defects creation in WGMs.

Electronic structure and processes of radiation damage in materials with complex chemical bonding and crystal structure is now under thorough and active investigation, both theoretical and experimental (see, e.g., [6, 10-12]).

\section{Experimental}

Nominally pure $\mathrm{Lu}_{3} \mathrm{Al}_{5} \mathrm{O}_{12}$ ( $\mathrm{LuAG}$, contains up to $30 \mathrm{ppm}$ of $\mathrm{Ce}^{3+}$ impurity ions) and LuAG: $\mathrm{Ce}^{3+}\left(\sim 700\right.$ or $1400 \mathrm{ppm}$ of $\left.\mathrm{Ce}^{3}\right)$ single crystals were grown from the melt by the Czochralski method under argon atmosphere containing hydrogen in CRYTUP, Turnov, Czech Republic. $\mathrm{MgO}$ single crystals were grown in Tartu from a highly pure starting 
material by a variation of the arc-fusion method. Nominally pure samples contain about 10 and 5 ppm of $\mathrm{Ca}^{2+}$ and $\mathrm{Cr}^{3+}$ impurity ions, respectively, while the concentration of $\mathrm{Cr}^{3+}$ ions in $\mathrm{MgO}: \mathrm{Cr}^{3+}$ equals 100 or $850 \mathrm{ppm}$. Freshly cleaved $\mathrm{MgO}$ crystals and polished plates of LuAG have been used for the investigations.

The crystals were irradiated with SHIs $\left({ }^{238} \mathrm{U},{ }^{197} \mathrm{Au},{ }^{209} \mathrm{Bi}, \sim 2.1 \mathrm{GeV}, 10^{12}\right.$ ions $\mathrm{cm}^{-2}$, range of 54-95 $\mu \mathrm{m}$ ) at room temperature at the UNILAC linear accelerator of the GSI, Darmstadt. The spectra of the induced optical absorption were measured in the spectral region of 6.5-1.5 eV using a high-absorbance spectrometer JASCO V-660 and in the region of 4.5-8.0 eV using a vacuum monochromator VMR-2. The spectra of fast cathodoluminescence were measured using single powerful electron pulses from the Koval'chuk-Mesyats type generator $\left(3 \mathrm{~ns}, 300 \mathrm{keV}\right.$, the current density 1-180 $\left.\mathrm{A} \mathrm{cm}^{-2}, 80 \mathrm{~K}\right)$. The photoluminescence experiments were carried out at $10 \mathrm{~K}$ using synchrotron radiation at the SUPERLUMI station of HASYLAB at DESY, Hamburg. The excitation spectra were normalized to equal the quantum intensities of the synchrotron radiation falling onto the crystal (a sodium salicylate was used as the reference signal). Time-integrated luminescence as well as the emission measured within a time window (length $\Delta t$ ) correlated with the excitation pulse (delayed by $\delta t$ ) were detected.

\section{Main mechanisms of radiation damage in wide-gap materials}

Insufficient radiation resistance is a serious limitation for WGMs possessing other necessary properties to be used as selective dosimeters for medical and industrial purposes, fast and effective scintillation detectors, spectral transformers of VUV-emission into visible light for environmentally friendly fluorescence lamps and displays, etc. No success in future industrial fission reactors can be achieved without a significant enhancement of the radiation resistance of different construction and functional materials. Accumulation of so-called longlived radiation defects, which remain stable for hours, days and months, plays a crucial role in material degradation under prolonged irradiation. However, the number of short-lived defects with lifetime $10^{-10}-10^{1} \mathrm{~s}$ is usually significantly higher.

By now it has been experimentally and theoretically proved that a number of mechanisms are responsible for radiation damage in WGMs (see, e.g. [1, 3-8]). First of all, a universal for solids knock-out (impact) mechanism is connected with elastic collisions of high-energy incident particles with the crystal nuclei, providing the displacement of atoms from their regular lattice sites into interstices with the formation of pairs of Frenkel defects (FDs) -vacancies (v) and interstitials (i). This rapid $\left(10^{-15} \mathrm{~s}\right)$ mechanism plays a dominant 
role in radiation damage of metals and alloys. In many ionic metal oxides, the formation energy (displacement energy) of an i-v pair, $E_{\mathrm{FD}}$ reaches several dozens of electron-volts [9].

Besides elastic collisions with nuclei, the absorbed energy is also spent on the excitation and ionization of an electron subsystem - that is the formation of different EEs. The nonradiative decay of these excitations into FD pairs is considered as the second creation mechanism [3-5]. The value $E_{\mathrm{FD}}$ for these relatively slow adiabatic mechanisms $\left(\sim 10^{-10} \mathrm{~s}\right)$, when system can select the optimized way of the energy transfer, is significantly lower than for the impact mechanism. A joint/simultaneous action of impact (elastic collisions) and nonimpact (with the participation of EEs) mechanisms leads to especially complicated creation processes of stable defects.

In different types of solids, intrinsic bulk EEs possess specific features that influence the creation of radiation defects. The criteria of the efficient decay of EEs into structural defects for model alkali halide crystals were formulated long ago [4, 7]. The first, energetic criterion is obvious: $E_{\mathrm{EE}}>E_{\mathrm{FD}}$. According to time criterion, the lifetime of an $\mathrm{EE}$ on a lattice site should exceed the period of effective lattice vibrations. It is worth noting, that besides creation, defects in a stable (long-lived) Frenkel pair should be spatially separated. Closely packed ion rows facilitate such separation via a crowdion motion (the so-called orientation criterion, see also [13]). The creation of a group (triplet) of spatially correlated point defects was detected in a number of alkali halides at the decay of a cation exciton (its formation energy is significantly higher than for an anion one) or of an EE with the energy exceeding $2 E_{\mathrm{g}}[14-16]$.

Based on the ratio between $E_{\mathrm{FD}}$ and $E_{\mathrm{g}}$, mainly ionic WGMs can be divided into two groups. In case of inequality $E_{\mathrm{FD}}<E_{\mathrm{g}}$, FDs are formed at the recombination of totally relaxed conduction electrons (e) and valence holes (h) and often even at the decay of self-trapping excitons. The latter mechanism is very efficient in many alkali halides [3-5, 7]. For many metal oxides $E_{\mathrm{FD}}>E_{\mathrm{g}}$, therefore, the energetic criterion for low-energy EEs is not fulfilled and the impact mechanism was expected to be the sole reason of radiation damage. However, if the process starts from a core EE with sufficiently long lifetime, the energy portion larger than $E_{\mathrm{FD}}$ can be obtained. FDs can be also formed at the recombination of nonrelaxed e and h, because the energy released at such hot e-h recombination can already exceed $E_{\mathrm{FD}}$ [17-20]. Usually a fraction of hot recombination is small, but recombination probability significantly increases under conditions of high-dense excitation.

The third complex creation mechanism of radiation damage was detected under superhigh density of EEs caused by high-current electron accelerators. Cracking and brittle destruction of single crystals at high-current electron pulses was explained by the generation 
of shock supersonic waves causing a local phase transition (see [21, 22] and references therein).

This third creation mechanism even more evidently manifests itself at the irradiation of WGMs with $E_{\mathrm{FD}}>E_{\mathrm{g}}$ by SHIs. In WGMs, SHIs with the energy of $\sim \mathrm{GeV}$ form cylindrical tracks with the length up to $100 \mu \mathrm{m}$ and spend more than $99.9 \%$ of their energy on the excitation of an electron subsystem thus providing a superhigh density of EEs along the tracks $[10,11,23-27]$. It is experimentally proved that radiation damage in ion-irradiated $\mathrm{MgO}$ is not limited by the impact mechanism of FDs creation, which is dominant under irradiation with fast neutrons. There is an obvious difference in the absorption and cathodoluminescence spectra of $\mathrm{MgO}$ preliminarily irradiated with fast neutrons or swift heavy ions [18-20]. Therefore, a joint non-additive action of the knock-out mechanism (especially efficient close to the track's end) and nonimpact (EE-related) mechanisms of defect creation should be considered under SHI-irradiation. At an ion track periphery, F-type colour centres are formed due to hot e-h recombination, while more complex and temperature-stable radiation defects are formed close to the track cores by collective excitations (thermal spike, Coulomb explosion, exciton plasma, etc.) [11]. Notice that fast (in a picosecond range) electron-hole plasma luminescence from track core was found in the majority of alkali halides, $\mathrm{MgO}$ and $\mathrm{Al}_{2} \mathrm{O}_{3}[28-29]$.

According to theoretical predictions, intrinsic discrete breathers (vibrational solitons) with frequency above the top of the phonon spectrum appear in metals and alloys under dense excitation (see $[30,31]$ and references therein). In WGMs, the frequency of discrete breathers can fall in a gap between acoustic and optical vibration branches. Discrete breathers possess high mobility and efficiently transfer concentrated vibrational energy over large distances in a crystal lattice. In metal oxides, such movement is expected along oxygen chains. A collapse of mobile breathers can occur in certain lattice regions with increased vibration amplitude and anharmonicity, e.g. when a breather meets some complex impurity centres [32]. The collapse causes a rearrangement of many host atoms, i.e. the formation of 3D nanosize defects stable up to a very high temperature. It is very difficult to anneal thermally such $3 \mathrm{D}$ cooperative defects. However, a simultaneous additional irradiation of the samples facilitates the annealing process [33]. Complex defects serve as stoppers for dislocations impeding their movement to the surface and facilitating the cracking and brittle destruction of irradiated single crystals. There are some preliminary manifestations of the similar effects in WGMs built up of ions with different masses, where anharmonicity is especially strong [34]. 


\section{Influence of impurity ions on the efficiency of defect creation}

There are no absolutely stoichiometric and impurity-free single crystals. Therefore, the contribution of impurity ions to radiation resistance is very important. Many WGMs used for applications are solid solutions of luminescent impurity ions, and their properties (including light yield and radiation resistance) depend on impurity concentration. In general, almost all impurities in the crystalline materials act as intensifiers (sometimes even as catalysts) or depressors for the creation of radiation defects. Furthermore, these effects strongly depend on the impurity type, including their charge state, concentration, distribution, etc. [35, 36].

A statistical distribution of application-useful ions is well known for the crystals with various structures. However, not all circumstances are usually taken into account. There is a significant energetic profit when two impurity ions undergo pairing (coupling) near anion or cation vacancies. Such benefit decreases the threshold for the formation of close impurity pairs in scintillators by many times. Similar to two- or many-atom molecules, a close impurity pair of heavy rare-earth ions $\mathrm{RE}^{3+}$ possesses complex adiabatic potentials. Therefore, anharmonic interactions result in the decrease of the temperature of luminescence quenching (thermal quenching) or even in thermal dissociation of such complex centres. The presence of close impurity pairs decreases both the efficiency of useful luminescence and the resistance of single crystals against radiation of different types.

Very stoichiometric and pure, with the content of impurities on the level of $1 \mathrm{ppm}$, facecentered cubic $\mathrm{MgO}$ crystals with $E_{\mathrm{FD}}>E_{\mathrm{g}}$ exhibit extremely high resistance against irradiation, even with $\sim \mathrm{GeV}$ heavy ions. It should be pointed out that the latter conclusion is valid only for a crystal bulk and not for near-surface layers, where the defect creation is facilitated (see, e.g., [37]). The presence of even $\sim 10 \mathrm{ppm}$ of light impurity ions $\left(\mathrm{Be}^{2+}, \mathrm{Ca}^{2+}\right)$ decreases the radiation resistance of $\mathrm{MgO}$ single crystals. Even these isovalent to $\mathrm{Mg}^{2+}$ impurity ions serve as efficient traps for radiation-induced holes and, therefore, increase the probability of a sequent recombination of hot conduction electrons with trapped-hole centres (hot $\mathrm{e}-\mathrm{h}$ recombination) resulting in the creation of FDs (see $[38,39]$ and references therein).

Several types of pair chromium centres have been already observed in $\mathrm{MgO}: \mathrm{Cr}$ single crystals $[1,2,34,40,41]$. In a tetragonal linear centre $\mathrm{Cr}^{3+}-\mathrm{O}^{2-}-\mathrm{V}_{\mathrm{Mg}}-\mathrm{O}^{2-}-\mathrm{Cr}^{3+}$, two heavy $\mathrm{Cr}^{3+}$ impurity ions (atomic mass 54, versus 24 for $\mathrm{Mg}$ ) substituting for $\mathrm{Mg}^{2+}$ are spatially separated from each other by oxygen ions and a cation vacancy $V_{M g}$ that is needed for charge compensation. Figure 1 presents two possible variants of pair chromium centres with rhombic symmetry, the number of which significantly increases with the rise of $\mathrm{Cr}^{3+}$ concentration from 100 to $850 \mathrm{ppm}$. A triangular centre with two spatially close $\mathrm{Cr}^{3+}$ and $\mathrm{V}_{\mathrm{Mg}}$ serves as an 
efficient trap for a radiation-induced hole at the oxygen ion depicted by a solid circle. To the right of Fig. 1 there is alternative, linear configuration of rhombic centre. During irradiation producing high density of e-h pairs, holes become trapped at rhombic centres, and the recombination of hot conduction electrons with trapped-hole centres takes place. The efficiency of recombination luminescence related to the mentioned hole-trapped centres is very low (fractions of percent) both at room and low temperatures. On the other hand, in case of $\mathrm{MgO}$ doping with light ${ }^{27} \mathrm{Al}^{3+}$ ions the yield of related recombination UV-luminescence is significantly higher, about $80 \%$. In our opinion, heavy two-chromium centres serve as centres of nonradiative recombination. Under irradiation with $\sim \mathrm{GeV}$-ions these recombinations partly results in the formation of structural defects including F-type centres and interstitials, bivacancies, F-aggregates, metal colloids, etc.

From radiation damage standpoint, one more complex two-chromium centre located nearby a $\mathrm{Ca}^{2+}$ impurity ion is of particular interest. For a large-size $\mathrm{Ca}^{2+}$ (electron configuration $3 s^{2} 3 p^{6} 3 d^{0}$ ) it is energy-profitable to be located nearby a bivacancy $\mathrm{V}_{\mathrm{Mg}} \mathrm{V}_{\mathrm{O}}$ within a complex centre. The optical characteristics of $\mathrm{V}_{\mathrm{Mg}} \mathrm{V}_{\mathrm{O}}$ (similar to those in alkali halide crystals [42]) were revealed in $\mathrm{MgO}$ crystals grown from the melt [27]. It was shown earlier that a low-temperature emission peaked at $6.8 \mathrm{eV}$ is related to the recombination of conduction electrons with the holes localized near $\mathrm{Ca}^{2+}$ in $\mathrm{MgO}: \mathrm{Ca}$ ( 100 ppm) [38].

The majority of wide-gap metal oxides are less radiation-resistant than $\mathrm{MgO}$. In principle, the radiation resistance of some WGMs can be enhanced by a light doping due to the socalled solid-state analogue of the Franck-Hertz effect revealed in alkali halide crystals doped with $\mathrm{Tl}^{+}$and $\mathrm{Ag}^{+}$impurity ions, which have both a ground and an excited state energy levels within an energy gap [43-45]. In some cases, a hot conduction electron can spent its energy excess for the direct excitation of an impurity centre up to the excited state, while a subsequent recombination of the cooled carriers already provides insufficient energy for the creation of a pair of FDs. The Franck-Hertz effect is considered as some kind of "luminescent protection" against radiation damage of WGMs, caused via hot e-h recombination [17, 39, 46].

Such "luminescent protection" takes place in some materials, for instance in $\mathrm{BaMgAl}_{10} \mathrm{O}_{17}: \mathrm{Eu}^{2+}$-phosphors with $\beta$-alumina structure [15]. A heavy impurity ${ }^{157} \mathrm{Eu}^{2+}$ (atomic mass 157) substitutes for a heavy ${ }^{152} \mathrm{Ba}$ ion located at the intermediate layer between two spinel blocks. It is important, that impurity ions are spatially separated from each other even in the case of high concentration. The luminescence efficiency of such single centres is high at the excitation by an electron beam or VUV-radiation. In addition, the phosphor is sufficiently resistant against radiation. 
The experimental manifestations of the above-mentioned luminescent protection have been also detected in $\mathrm{Lu}_{3} \mathrm{Al}_{5} \mathrm{O}_{12}(\mathrm{LuAG})$ single crystals with a relatively low concentration of $\mathrm{Ce}^{3+}$ impurity ions, which are mainly located at $\mathrm{Lu}^{3+}$-sites $\left(\mathrm{Ce} \mathrm{Lu}_{\mathrm{Lu}}\right.$ ) (see also [39]). The spectra of radiation-induced optical absorption have been compared for nominally pure and purposely Ce-doped crystals after their isodose irradiation with ${ }^{197} \mathrm{Au}$ ions $\left(2.04 \mathrm{GeV}, 10^{12} \mathrm{Au} / \mathrm{cm}^{2}, 300\right.$ $\mathrm{K})$. The presence of $\mathrm{Ce}^{3+}$ ions causes some decrease of induced optical absorption at 5.2-6.1 $\mathrm{eV}$ partly related to F-type centres. However, at the rise of cerium concentration up to $\sim 1-2 \%$ that is needed for the pronounced "luminescent protection", the situation becomes more complicated due to the formation of pair impurity centres (see also Section 5).

Furthermore, the achieved protection in $\mathrm{LuAG}: \mathrm{Ce}^{3+}$ is rather weak compared to the wellknown case of $\mathrm{Y}_{3} \mathrm{Al}_{5} \mathrm{O}_{12}: \mathrm{Nd}^{3+}$ - an extremely powerful and resistant neodymium laser. During irradiation, $\mathrm{Nd}^{3+}$ impurity ions gain a significant part of the absorbed energy and transform it into infrared luminescence in the region of matrix transparency, thus avoiding dangerous heating of a crystal due to possible luminescence reabsorption. Similar situation can probably be realized in $\mathrm{Lu}_{3} \mathrm{Al}_{5} \mathrm{O}_{12}: \mathrm{Nd}^{3}$ scintillators a well. The important example of luminescent protection against SHI-induced radiation damage was reported earlier for a CsI:Tl classical scintillator with bec lattice [47].

It is worth of notice that, in general, luminescent protection can increase the radiation resistance of even slightly doped WGMs with $E_{\mathrm{FD}}>E_{\mathrm{g}}$, if the value of $E_{\mathrm{FD}}$ for the material does not exceed $2 E_{\mathrm{g}}$. A photon with the energy of $\sim 2 E_{\mathrm{g}}$ creates a hot conduction electron (an energy excess of $E_{\mathrm{g}}$ ) that already participates in the so-called multiplication process forming a secondary e-h pair (see, e.g., [43, 45]), while both existing conduction electrons become "cold" for the direct excitation of an impurity centre.

\section{Intrinsic and impurity antisite defects in lutetium garnet single crystals}

LuAG: $\mathrm{Ce}^{3+}$ single crystals are very promising for applications due to high density (6.67 $\mathrm{g} \mathrm{cm}^{-3}$ ), assumed high resistance against irradiation (belongs to WGMs with $E_{\mathrm{FD}}>E_{\mathrm{g}}$ ), good mechanical and chemical stability, reasonable scintillation parameters ( $\sim 60 \mathrm{~ns}, \sim 26000$ photons $\mathrm{MeV}^{-1}$ ), etc. Isostructural to $\mathrm{Y}_{3} \mathrm{Al}_{5} \mathrm{O}_{12} \mathrm{LuAG}$ single crystals have bcc cubic structure with space group Ia3d. An elementary cell contains 160 atoms: $\mathrm{Al}^{3+}$ ions are located in lattice sites with octahedral (16 sites) or tetrahedral (24) oxygen coordination, $\mathrm{Lu}^{3+}$ ions are placed in 24 dodecahedral sites. However, lutetium nucleus is about twice as heavy as an yttrium one, consists of 71 protons and 104 neutrons (for the main isotope, 97.4\%), and nuclear spin of $\mathrm{Lu}$ significantly exceeds that for yttrium ( $7 / 2$ and $1 / 2$, respectively [48]). 
Three series of LuAG single crystals with the $\mathrm{Ce}^{3+}$ impurity concentration of $\sim 30 \mathrm{ppm}$ (nominally pure sample), 700 or $1400 \mathrm{ppm}$ have been investigated in order to analyse the complex process of radiation damage under irradiation with SHIs $\left({ }^{238} \mathrm{U},{ }^{197} \mathrm{Au},{ }^{209} \mathrm{Bi}, \sim 2.1\right.$ $\mathrm{GeV}, 10^{12}$ ions $\mathrm{cm}^{-2}$ ) at room temperature (see, e.g. [24, 26] for details of irradiation). At the irradiation fluence used, the average distance between centres of two neighbour ion tracks in LuAG is about $11 \mathrm{~nm}$. The present paper is a continuation of our investigations of SHIinduced radiation damage in LuAG [34, 39].

It was experimentally proved that small amount of $\mathrm{Lu}^{3+}$ ions can reside in the $\mathrm{Al}^{3+}$ octahedral positions forming the so-called antisite defect $\left.\mathrm{Lu}\right|_{\mathrm{Al}}-$ an ion in a "wrong" cation position (see, e.g., [49-52]). The $\sim 4.5 \mathrm{eV}$ emission with the decay time typical of triplet (exciton-like) states was associated with $\left.\mathrm{Lu}\right|_{\mathrm{Al}}$. This interpretation became generally recognized after the detection of the strong attenuation of the $\sim 4.5 \mathrm{eV}$ emission in $\mathrm{LuAG}$ single crystalline films grown at temperature significantly below the melting point and containing, as a consequence, the reduced amount of $\left.\left.\mathrm{Lu}\right|_{\mathrm{Al}}[53-55]\right)$. The wide emission band peaked at $\sim 5 \mathrm{eV}$ is ascribed to small-radius oxygen-related excitons in a virgin LuAG crystal (see [39] and references therein). Irradiation of a crystal with SHIs weakens this emission by a factor of $\sim 5$. Interconfiguration electron transitions $\mathrm{f}^{14} \rightarrow \mathrm{f}^{13} \mathrm{~d}^{1}$ in $\mathrm{Lu}^{3+}$ in a vacuum ultraviolet spectral region (zero-phonon lines at 81760 and $83150 \mathrm{~cm}^{-1}$ ) that means the formation of a lutetium cation exciton have been revealed for $\mathrm{LiLuF}_{4}$ crystals [56]. In LuAG, a cation exciton is formed in the same region, slightly below $11 \mathrm{eV}$ [39].

The creation of $\mathrm{F}$ and $\mathrm{F}^{+}$centres (two or one electrons trapped by an oxygen vacancy) was detected at periphery of cylindrical tracks of SHIs in LuAG single crystals using highly sensitive luminescent methods [34, 39]. It has also been suggested than the collapse of mobile discrete breathers in certain lattice places causes the creation of complex defects involving many host atoms [34]. In a bulk of wide-gap metal oxides with oriented covalent bonds and a complicated shape of interstitial space, the formation of single interstitial atoms (as a part of a Frenkel pair) is impeded. According to theoretical estimation [57], the formation energy for an antisite defect in LuAG is significantly lower than that for a pair of FDs. Therefore, it is easier to replace a light matrix metal ion for a heavy one thus forming intrinsic antisite defects $\left(\left.\mathrm{Lu}\right|_{\mathrm{Al}}\right)$, where the radiation-induced EEs will be efficiently formed.

Fig. 2 presents the excitation band at $6.9-7.3 \mathrm{eV}$ measured for $\left.\mathrm{Lu}\right|_{\mathrm{Al}}-$ related emission in a virgin sample of a nominally pure LuAG at $10 \mathrm{~K}$. This result confirms the presence of a relatively small amount of single as-grown $\left.\mathrm{Lu}\right|_{\mathrm{Al}}$ defects. The efficient creation of $\left.\mathrm{Lu}\right|_{\mathrm{Al}}$ takes place under irradiation of LuAG with SHIs providing an extremely high density of EEs within 
ion tracks $(L E T>20 \mathrm{keV} / \mathrm{nm}$, ion range $\sim 50 \mu \mathrm{m})$ and a joint action of nonimpact and knockout mechanisms of defect creation. Incident SHIs as well as primary and secondary knock-on lutetium and aluminium (especially from octahedral configuration) ions participate in the formation of $\left.\mathrm{Lu}\right|_{\mathrm{Al}}$, while EEs recharge complex defects and provide additional creation of antisite defects. $\left.\mathrm{Lu}\right|_{\mathrm{Al}}$ is created under conditions of local heating that, probably, enhances its magnetic interaction with a neighbour $\mathrm{Lu}$ in a regular cation site. As a result, spatially close $\left.\mathrm{Lu}\right|_{\mathrm{Al}}$ and $\left.\mathrm{Lu}\right|_{\mathrm{Lu}}$ (distance less than $0.3 \mathrm{~nm}$ ), formed at the first stage of dense irradiation, serve as a place for the further collapse of a discrete breather. In our opinion, strong magnetic interaction between lutetium nuclei ( $\operatorname{spin} 7 / 2$ ) can be responsible for significantly lower radiation resistance of $\mathrm{LuAG}$ with respect to $\mathrm{Y}_{3} \mathrm{Al}_{5} \mathrm{O}_{12}$ ( $\mathrm{Y}$ nuclear spin is 1/2).

The optical characteristics of radiation-induced antisite defects differ from those for single as-grown $\left.\mathrm{Lu}\right|_{\mathrm{Al}}$. The excitation spectrum measured at $10 \mathrm{~K}$ for the $4.5 \mathrm{eV}$ emission in a SHI-irradiated LuAG contains a well-pronounced band peaked at $\sim 8.0 \mathrm{eV}$, which can be tentatively attributed to radiation-induced associations of $\left.\mathrm{Lu}\right|_{\mathrm{Al}}$ defects (for instance, $\left.\mathrm{Lu}\right|_{\mathrm{Al}}-\left.\mathrm{Lu}\right|_{\mathrm{Al}}$ pairs). Such complex structure possesses strong anharmonicity providing an increased probability of nonradiative transitions (with heat release or the creation of structural defects) from the excited state of a $\left.\mathrm{Lu}\right|_{\mathrm{Al}}-\left.\mathrm{Lu}\right|_{\mathrm{Al}}$ centre. It is worth noting that all emission efficiencies (incl. Lu$\left.\right|_{\mathrm{Al}}$-related one) are significantly attenuated in the SHI-irradiated sample, partly due to a radiation-induced optical absorption and the corresponding effects of luminescence reabsorption.

The manifestations of radiation-induced defects can be also revealed at the comparison of reflection spectra measured at $10 \mathrm{~K}$ for SHI-irradiated and virgin LuAG crystals. According to Ref. [39], the ratio of these reflection spectra (irradiated/virgin) contains a pronounced peak at $\sim 7.1 \mathrm{eV}$ and a small peculiarity at $\sim 8.0 \mathrm{eV}$, that, in our opinion, confirm the formation of single and more complex (pair) $\left.\mathrm{Lu}\right|_{\mathrm{Al}}$ defects. Our recent measurements have shown that the thermal annealing of the $\sim 7 \mathrm{eV}$-band of SHI-induced optical absorption efficiently occurs above $1100 \mathrm{~K}$ (at about a half of the melting point), where the intensive ionic movement takes place already.

The appearance of radiation damage in SHI-irradiated crystals can be demonstrated even comparing the excitation spectra measured for the time-integrated $\mathrm{Ce}^{3+}$ emission $(2.46 \mathrm{eV})$ in virgin and SHI-irradiated samples LuAG:Ce ${ }^{3+}(1400 \mathrm{ppm})$ using synchrotron radiation at $10 \mathrm{~K}$ (see Fig. 2). In the irradiated crystal, the cerium emission efficiency is strongly decreased, especially at 5.7-7.8 eV. The dashed line demonstrates the difference between the excitation spectra arbitrary normalized at the low-energy maximum. The difference curve contains two 
pronounced maxima at $\sim 6$ and $\sim 7 \mathrm{eV}$ connected with the increased absorption of radiationinduced F-type and $\left.\mathrm{Lu}\right|_{\mathrm{Al}}$ defects.

It has already been mentioned that nominally pure LuAG crystals contain up to $30 \mathrm{ppm}$ of $\mathrm{Ce}^{3+}$ impurity ions that usually substitute for $\mathrm{Lu}^{3+}\left(\left.\mathrm{Ce}\right|_{\mathrm{Lu}}\right)$. Based on a comparison of the EPR spectra related to $\mathrm{Ce}^{3+}$ in $\mathrm{LuAG}: \mathrm{Ce}^{3+}$ single crystals with different cerium concentrations, it was stated that there also exists the so-called impurity antisite defect $\left.\mathrm{Ce}\right|_{\mathrm{Al}}-\mathrm{a} \mathrm{Ce}^{3+}$ ion located in an octahedral $\mathrm{Al}^{3+}$ site [52]. Because of the larger ionic radius of $\mathrm{Ce}^{3+}$ ion as compared with $\mathrm{Lu}^{3+},\left.\mathrm{Ce}\right|_{\mathrm{Al}}$ causes a larger distortion of a crystal lattice than intrinsic antisite defect $\left.\mathrm{Lu}\right|_{\mathrm{Al}}$. The formation of $\left.\mathrm{Ce}\right|_{\mathrm{Al}}$ is even more favourable near an anion vacancy, and $\left.\mathrm{Ce}\right|_{\mathrm{Al}}$ nearby the F-type defects were also revealed in LuAG using the EPR method [52].

The emission spectra (pulse amplitude) at the excitation of virgin and ion-irradiated LuAG crystals $\left(30 \mathrm{ppm}\right.$ of $\left.\mathrm{Ce}^{3+}\right)$ by single powerful electron pulses $(300 \mathrm{keV}, 3 \mathrm{~ns}, 80 \mathrm{~K})$ have been analysed in Ref. [39]. Besides $\mathrm{Ce}^{3+}$ luminescence $\left(\sim 2.4 \mathrm{eV},\left.\mathrm{Ce}\right|_{\mathrm{Lu}}\right)$, the emissions related to self-trapped excitons $(\sim 5.1 \mathrm{eV})$ and $\left.\mathrm{Lu}\right|_{\mathrm{Al}}(\sim 4.5 \mathrm{eV})$ were distinguished in the spectrum for a virgin LuAG. In SHI-irradiated LuAG, the enhancement of the emission peak amplitude was detected only at $3.25-4.25 \mathrm{eV}$. The absolute enhancement of this emission at $\sim 3.75 \mathrm{eV}$ of unknown origin was detected due to its short decay time ( $70 \mathrm{~ns})$ at $80 \mathrm{~K}$ [39]. This $3.75 \mathrm{eV}$-emission complex band is reproduced in Fig. 3. According to our additional experiments, the emission undergoes reversible thermal quenching (see insert in Fig. 2) typical for intracentre luminescence. The emission is detectable only from the crystal side previously irradiated by SHI and, in our opinion, is connected with single impurity antisite defects $\left.\mathrm{Ce}\right|_{\mathrm{Al}}$, the number of which significantly increases under SHI-irradiation.

Although single $\left.\mathrm{Ce}\right|_{\mathrm{Lu}}$ dominate in slightly doped virgin LuAG crystals, the number of $\left.\mathrm{Ce}\right|_{\mathrm{Lu}}-\left.\mathrm{Ce}\right|_{\mathrm{Lu}}$ pairs increases with the rise of cerium concentration. The number of impurity antisite defects $\left.\mathrm{Ce}\right|_{\mathrm{Al}}$ can be also significantly increased due to irradiation with SHIs. The increased number of $\left.\mathrm{Ce}\right|_{\mathrm{Al}}$ can cause the decrease in the radiation resistance of LuAG. In a pair of spatially close heavy cerium ions, one of which substitutes for a light octahedral aluminium ion $-\left.\mathrm{Ce}\right|_{\mathrm{Al}}$ and another is located in a regular lutetium position $\left(\left.\mathrm{Ce}\right|_{\mathrm{Lu}}\right)$, a strong local anharmonicity arises. Such impurity pair $\left.\mathrm{Ce}\right|_{\mathrm{Lu}}-\left.\mathrm{Ce}\right|_{\mathrm{Al}}$ can serve as a place for the collapse of a mobile breather resulting in the creation of 3D collective defects under dense irradiation with $L E T>20 \mathrm{keV} / \mathrm{nm}$. In a $\left.\mathrm{Ce}\right|_{\mathrm{Lu}}-\left.\mathrm{Ce}\right|_{\mathrm{Al}}$ quasi-molecular complex, the distance between $\mathrm{Ce}^{3+}$ ions is about $0.3 \mathrm{~nm}$, i.e. about a half of the distance between regular lutetium sites in a crystal lattice. In order to compensate the space deficit for two neighbour large-radius $\mathrm{Ce}^{3+}$ ions, an oxygen vacancy can be located somewhere in their vicinity. 
Fig. 3 shows the emission spectra detected for a time-integrated signal as well as for the emission detected within a time window (delayed by $\delta t=20 \mathrm{~ns}$, length $\Delta t=30 \mathrm{~ns}$,) at the excitation of LuAG: $\mathrm{Ce}^{3+}(1400 \mathrm{ppm})$ with $6.1 \mathrm{eV}$ photons at $10 \mathrm{~K}$. Besides the emission of regular $\mathrm{Ce}^{3+}$ ions $\left(\left.\mathrm{Ce}\right|_{\mathrm{Lu}}\right.$, maxima at 2.22 and $\left.2.46 \mathrm{eV}\right)$, the spectrum contains an emission band peaked at $\sim 3.2 \mathrm{eV}$. The emission maximum of $\mathrm{F}^{+}$centres lies in the same spectral region. However, according to Ref. [55], the decay time for $\mathrm{F}^{+}$-emission in LuAG at low temperature is about $3 \mathrm{~ns}$, while no emission in a short time window ( $\delta t=2 \mathrm{~ns}, \Delta t=5 \mathrm{~ns}$ ) was detected in our experiment. Based on the analysis of our and literature data, we tentatively ascribe the emission peaked at $\sim 3.2 \mathrm{eV}$ to an impurity pair $\left.\mathrm{Ce}\right|_{\mathrm{Lu}}-\left.\mathrm{Ce}\right|_{\mathrm{Al}}$. Earlier the variety of $\left.\mathrm{Ce}\right|_{\mathrm{Al}}$ defects, single or disturbed by different defects/impurities were detected and thoroughly investigated by the EPR and optical methods (see, e.g. [55] and references therein).

A further complex experimental study of the influence of complex (pair) centres on the efficiency of radiation defect creation still lies ahead. Particular emphasis should be laid to the above-mentioned close cation pairs $\left.\mathrm{Lu}\right|_{\mathrm{Al}}-\left.\mathrm{Lu}\right|_{\mathrm{Lu}}$ with strong magnetic interaction between lutetium nuclei (spin 7/2, separation between nuclei about $0.3 \mathrm{~nm}$ ) that tentatively facilitates their mutual approach, changes the ground and excited electronic states as well as transition probabilities in two-lutetium centres. As far as we know, such magnetic interaction between lutetium nuclei in LuAG has not been considered in the literature yet.

It is worth noting, that the radiation damage of construction and functional materials in nuclear reactors (including advanced ones) can be emulated by irradiation with swift light (protons) and heavy ions (see recent review article [58]). Comparing to fast neutron irradiation, ion-irradiation has a very low cost, excellent control over irradiation conditions and can provide in a reasonable time the damage up to $200 \mathrm{dpa}$ (construction materials should survive up to such damage). On the other hand, several additional factors should be taken into account for correct comparison of the damage caused by reactor- or ion-irradiation [58]. In our investigations, ${ }^{197} \mathrm{Au}$ or ${ }^{238} \mathrm{U}$ ions with zero nuclear spin and with the kinetic energy of $\sim 2 \mathrm{GeV}$ were usually used in order to analyse the mechanisms of radiation damage in WGMs. Recently a set of WGMs was irradiated at the UNILAC linear accelerator by $\sim 2 \mathrm{GeV}{ }^{209} \mathrm{Bi}$ ions with an extremely high nuclear spin of $9 / 2$ [48]. According to our preliminary results, the isodose irradiation of $\mathrm{LuAG}$ and $\mathrm{Gd}_{2} \mathrm{SiO}_{5}$ with $\mathrm{Bi}$ ions causes more efficient cracking of single crystals than in the case of $\mathrm{Au}$ or $\mathrm{U}$ ions. A further investigation lies ahead to confirm that this difference in radiation damage is partly connected with magnetic properties of $\mathrm{Bi}$ nuclei. 


\section{Concluding remarks}

Radiation damage in a bulk of many alkali halide crystals can be mainly explained by the creation of Frenkel pairs and their associations. In a bulk of complex (e.g., two-cation) wide-gap metal oxides with ionic-covalent bonds and a complicated shape of interstitial space, the formation of single interstitial atoms is impeded. It is easier to replace a light matrix metal with a heavy one and to form an intrinsic antisite defect ( $\left.\mathrm{Lu}\right|_{\mathrm{Al}}$ in $\left.\mathrm{LuAG}\right)$ where the radiationinduced EEs will be efficiently formed.

Considering complicated processes of stable defects creation by $\sim \mathrm{GeV}$ SHIs, a joint/simultaneous action of impact (knock-out) and nonimpact mechanisms related to the decay of different EEs should be taken into account. Under SHI-irradiation, it is energetically profitable to create defects via complex mechanisms - for instance, the collapse of mobile discrete breathers at complex impurity centres or defects (single or pair $\left.\mathrm{Lu}\right|_{\mathrm{Al}}$ ) thus providing local rearrangement of many host atoms - creation of complex radiation defects. Such nanosize 3D defects serve as stoppers for dislocations and facilitate the cracking and brittle destruction of irradiated single crystals. The considered complex mechanism of radiation damage was suggested in 1970-ies in Tomsk, Russia [21] and was later supported by theorists $[30,31]$.

The presence of impurity ions influences the efficiency of radiation damage of WGMs in a dual way. Light doping of some WGMs with $E_{\mathrm{FD}}>E_{\mathrm{g}}$ provides some kind of a "luminescent protection" against defect creation via hot e-h recombination, which is efficient under highdense excitation. However, several limitations do not allow to increase significantly radiation resistance of WGMs. The main one is connected with the formation of pair/complex-impurity centres that, because of high anharmonicity, can serve as the places for breathers collapse under dense irradiation. $\mathrm{Cr}^{3+}-\mathrm{Cr}^{3+}$ in $\mathrm{MgO}$ and $\mathrm{Ce}||_{\mathrm{Lu}}-\left.\mathrm{Ce}\right|_{\mathrm{Al}}$ in $\mathrm{LuAG}$ are considered as the examples of such complex impurity centres.

\section{Acknowledgements}

We are grateful to Drs. A. Shugai and S. Zazubovich for useful discussions. The research leading to these results has received funding from the Estonian Research Council Institutional Research Fundings IUT02-26 and European Community - through a joint programme of the members of the EUROfusion consortium (Horizon 2020 research and innovation programme under grant agreement number 633053). The views and opinions 
expressed herein do not necessarily reflect those of the European Commission. AIP thanks ESF grant No. 2013/0046/1DP/1.1.1.2.0/13/APIA/VIAA/ 021.

\section{References}

[1] M. Thompson, Defects and Radiation Damage in Metals, Univ. Press, Cambridge, 1969.

[2] B. Henderson, E. Wertz, Defects in the Alkaline Earth Oxides, Taylor \& Francis, London, 1977.

[3] Ch.B. Lushchik, in: R.A. Johnson, A.N. Orlov (Eds.), Physics of Radiation Effects in Crystals, North-Holland, Amsterdam, 1986 (Chapter 8). doi:10.1016/B978-0-444-869463.50014-0

[4] Ch.B. Lushchik, A.Ch. Lushchik, Decay of Electronic Excitations with Defect Formation in Solids, Nauka, Moscow, 1989.

[5] K.S. Song, R.T. Williams, Self-Trapped Excitons. Second ed., Springer, Berlin, 1996.

[6] N. Itoh, A.M. Stoneham, Material Modification by Electronic Excitation, Univ. Press, Cambridge, 2000.

[7] A. Lushchik, M. Kirm, Ch. Lushchik, E. Vasil'chenko, Nucl. Instrum. Methods B 166167 (2000) 529.

[8] E.A. Kotomin, A.I. Popov, in: K.E. Sickaufus, E.A. Kotomin, B.P. Uberuaga (Eds.) Radiation Effects in Solids, Book Series: NATO Science Series II-Mathematics Physics and Chemistry, vol. 235, 2007 (Chapter 7)

[9] A.I. Popov, E.A. Kotomin, J. Maier, Nucl. Instrum. Methods B 268 (2010) 3084.

[10] F.F. Komarov, Phys. Usp. 46 (2003) 1253.

[11] N. Itoh, D.M. Duffy, S. Khakshouri, A.M. Stoneham, J. Phys.: Condens. Matter 21 (2009) 474205.

[12] I. Kudryavtseva, M. Klopov, A. Lushchik, Ch. Lushchik, A. Maaroos, A. Pishtshev, Phys. Scr. 89 (2014) 044013.

[13] A.Ch. Lushchik, A.G. Frorip, Phys. Status Solidi B 161 (1990) 525.

[14] A. Lushchik, I. Kudryavtseva, Ch. Lushchik, E. Vasil'chenko, M. Kirm, I. Martinson, Phys. Rev. B 52 (1995) 10069.

[15] M. Kirm, A. Lushchik, Ch. Lushchik, Phys. Status Solidi A 202 (2005) 213.

[16] S. Nakonechnyi, T. Kärner, A. Lushchik, Ch. Lushchik, V. Babin, E. Feldbach, I. Kudryavtseva, P. Liblik, L. Pung, E. Vasil'chenko, J. Phys: Condens. Matter 18 (2006) 379. 
[17] A. Lushchik, Ch. Lushchik, M. Kirm, V. Nagirnyi, F. Savikhin, E. Vasil'chenko, Nucl. Instrum. Methods B 250 (2006) 330.

[18] A. Lushchik, T. Kärner, Ch. Lushchik, E. Vasil'chenko, S. Dolgov, V. Issahhanyan, P. Liblik, Phys. Status Solidi C 4 (2007) 1084.

[19] A. Lushchik, E. Feldbach, S. Galajev, T. Kärner, P. Liblik, Ch. Lushchik, A. Maaroos, V. Nagirnyi, E. Vasil'chenko, Radiat. Meas. 42 (2007) 792.

[20] A. Lushchik, Ch. Lushchik, K. Schwartz, E. Vasil'chenko, T. Kärner, I. Kudryavtseva, V. Isakhanyan, A. Shugai, Nucl. Instrum. Methods B 266 (2008) 2868.

[21] D.I. Vaisburd, I.N. Balychev, Sov. Phys. JETP 15 (1972) 380.

[22] D.I. Vaisburd, K.E. Evdokimov, Phys. Status Solidi C 2 (2005) 216.

[23] M. Toulemonde, S. Bouffard, F. Studer, Nucl. Instrum. Methods B 91 (1994) 108.

[24] K. Schwartz, G. Wirth, C. Trautmann, T. Steckenreiter, Phys. Rev. B, 56 (1997) 10711.

[25] V.A. Skuratov, S.J. Zinkle, A.E. Efimov, K. Havancsak, Nucl. Instrum. Methods B 203 (2003) 136.

[26] K. Schwartz, A.E. Volkov, M.V. Sorokin, R. Neumann, C. Trautmann, Phys. Rev. 82 (2010) 144116.

[27] A. Lushchik, Ch. Lushchik, K. Schwartz, F. Savikhin, E. Shablonin, A. Shugai, E. Vasil'chenko, Nucl. Instrum. Methods B 277 (2012) 40.

[28] K. Kimura, S. Sharma, A.I. Popov, Radiat. Meas. 34 (2001) 99.

[29] K. Kimura, S. Sharma, A.I. Popov, Nucl. Instrum. Methods B, 191 (2002) 48.

[30] M. Haas, V. Hizhnyakov, A. Shelkan, M. Klopov, A.J. Sievers, Phys. Rev. B 84 (2011) 144303.

[31] V. Hizhnyakov, M. Haas, A. Shelkan, M. Klopov. Phys. Scr. 89 (2014) 044003.

[32] V.E. Zakharov, E.A. Kuznetsov, Phys. Usp. 55 (2012) 535.

[33] V.V. Ovchinnikov, Phys. Usp. 51 (2008) 955.

[34] A. Lushchik, Ch. Lushchik, V. Nagirnyi, S. Pazylbek, O. Sidletskiy, K. Schwartz, E. Shablonin, A. Shugai, E. Vasil'chenko, Phys. Status Solidi B 250 (2013) 261.

[35] F. Agullo-Lopez, F.J. Lopez, F. Jaque, Crystal Lattice Defects 9 (1982) 227.

[36] M. Hirai, J. Phys. Chem. Solids 51 (1990) 737.

[37] P.V. Sushko, A.L Shluger, C.R. Catlow, Surf. Sci. 450 (2000) 153.

[38] S. Dolgov, T. Kärner, A. Lushchik, A. Maaroos, S. Nakonechnyi, E. Shablonin, Fiz. Tverd. Tela 53 (2011) 1179 [Phys. Solid State, 53 (2011) 1244].

[39] A. Lushchik, T. Kärner, Ch. Lushchik, K. Schwartz, F. Savikhin, E. Shablonin, A. Shugai, E. Vasil'chenko, Nucl. Instrum. Methods B, 286 (2012) 200.

[40] B. Henderson, CRC Crit. Rev. Solis State Mater. Sci. 9 (1980) 1. 
[41] G.F. Imbusch, A.L. Schawlow, A.D. May, S. Sugano, Phys. Rev. 140 (1965) A830.

[42] A. Lushchik, Ch. Lushchik, N. Lushchik, A. Frorip, O. Nikiforova, Phys. Status Solidi B 168 (1991) 413.

[43] E. Feldbach, M. Kamada, M. Kirm, A. Lushchik, Ch. Lushchik, I. Martinson, Phys. Rev. B 56 (1997) 13908.

[44] A. Lushchik, M. Kamada, M. Kirm, Ch. Lushchik, I. Martinson, Radiat. Meas., 29 (1998) 229.

[45] A. Lushchik, Ch. Lushchik, T. Kärner, P. Liblik, V. Nagirnyi, E. Shablonin, A. Shugai, E. Vasil'chenko, Radiat. Meas. 45 (2010) 268.

[46] A. Lushchik, Ch. Lushchik, P. Liblik, A. Maaroos, V. N. Makhov, F. Savikhin, E. Vasil'chenko, J. Lumin. 129 (2009) 1894.

[47] A.I. Popov, E. Balanzat, Nucl. Instrum. Methods B 166-167 (2000) 545.

[48] S.E. Frisch. Optical Atom Spectra. Second ed., Lan, Saint-Petersburg, 2010 (in Russian).

[49] M. Nikl, E. Mihokova, J. Peichal, A. Vedda, Yu. Zorenko, K. Nejezchleb, Phys. Status Solidi B 242 (2005) R119.

[50] M. Nikl, A. Vedda, M. Fasoli, I. Fontana, V.V. Laguta, E. Mihokova, J. Pejchal, J. Rosa, K. Nejezchleb, Phys. Rev. B 76 (2007) 195121.

[51] M. Nikl, V.V. Laguta, A. Vedda, Phys. Status Solidi B 245 (2008) 1701.

[52] V. Babin, V.V. Laguta, A. Makhov, K. Nejezchleb, M. Nikl, S. Zazubovich, EEE Trans. Nucl. Sci. 55 (2008) 1156.

[53] Y. Zorenko, V. Gorbenko, E. Mihokova, M. Nikl, K. Nejezchle, A. Vedda, V. Kolobanov, D. Spassky, Radiat. Meas. 42 (2007) 521.

[54] Y. Zorenko, T. Zorenko, V. Gorbenko, B. Pavlyka, V. Laguta, M. Nikl, V. Kolobanov, D. Spassky, Radiat. Meas. 45 (2010) 409.

[55] V. Babin, V. Bichevin, V. Gorbenko, M. Kink, A. Makhov, Yu. Maksimov, M. Nikl, G. Stryganyuk, S. Zazubovich, Yu. Zorenko, Phys. Status Solidi B 248 (2011) 1505.

[56] M. Kirm, G. Stryganyuk, S. Vielhauer, G. Zimmerer, V.N. Makhov, B.Z. Malkin, O.V. Solovyev, R.Yu. Abdulsabirov, S.L. Korableva, Phys. Rev. B, 75 (2007) 075111.

[57] M.M. Kuklja. J. Phys.: Condens. Matter 12 (2000) 2953.

[58] G.S. Was, J. Mater. Res. 30 (2015) doi:10.1557/jmr.2015.73 


\section{Captions}

Figure 1. Possible configurations of two-chromium rhombic centres in $\mathrm{MgO}$. In case of a triangular centre (in the left), a radiation-induced hole becomes trapped at the oxygen ion depicted by a solid circle.

Figure 2. Excitation spectra measured for the 2.46-eV emission of $\mathrm{Ce}^{3+}$ centres in a virgin (curve 1) and ion-irradiated (curve 2, multiplied by a factor of 8) $\mathrm{Lu}_{3} \mathrm{Al}_{5} 0_{12}: \mathrm{Ce}^{3+}(1400 \mathrm{ppm}$ ) single crystals at $10 \mathrm{~K}$. Curve 3 is obtained by a subtraction of curve 2 from curve 1 after their arbitrary normalization at the low-energy maximum. The excitation spectra measured for the 4.5 emission in a virgin (curve 4) and ion-irradiated (curve 5) LuAG single crystals at $10 \mathrm{~K}$. The crystals were irradiated by uranium ions $\left(2.14 \mathrm{GeV}, 10^{12}\right.$ ions $\left.\mathrm{cm}^{-2}\right)$ at $300 \mathrm{~K}$.

Fig. 3. The enhancement of emission peak amplitude (curve 1) at the excitation by single electron pulses $\left(300 \mathrm{keV}, 3 \mathrm{~ns}, 80 \mathrm{~A} \mathrm{~cm}^{-2}, 80 \mathrm{~K}\right)$ and temperature dependence of the 3.75-eV emission (curve 2) measured for an ion-irradiated $\mathrm{Lu}_{3} \mathrm{Al}_{5} \mathrm{O}_{12}$ crystal $\left(2.14 \mathrm{GeV}, 10^{12}\right.$ ions cm $\mathrm{cm}^{-2}$, $300 \mathrm{~K}$ ). The emission spectra measured for a time-integrated signal (curve 3) or the emission within a time window ( $\delta t=20 \mathrm{~ns}, \Delta t=30 \mathrm{~ns}$, curve 4$)$ at the excitation of LuAG:Ce ${ }^{3+}(1400$ ppm) with $6.1 \mathrm{eV}$ photons at $10 \mathrm{~K}$.

\section{Manuscript lengths}

8500 characters (with spaces) per a journal page. 1 single-column figure equals 0.15 pages. Manuscript: text in total (incl. abstract, references, captions) 43850 char. -5.15 pages + Abstract (empty space) $0,25+$ figs 0,45 . Totally $\sim \mathbf{5 . 8 5}$ journal pages 


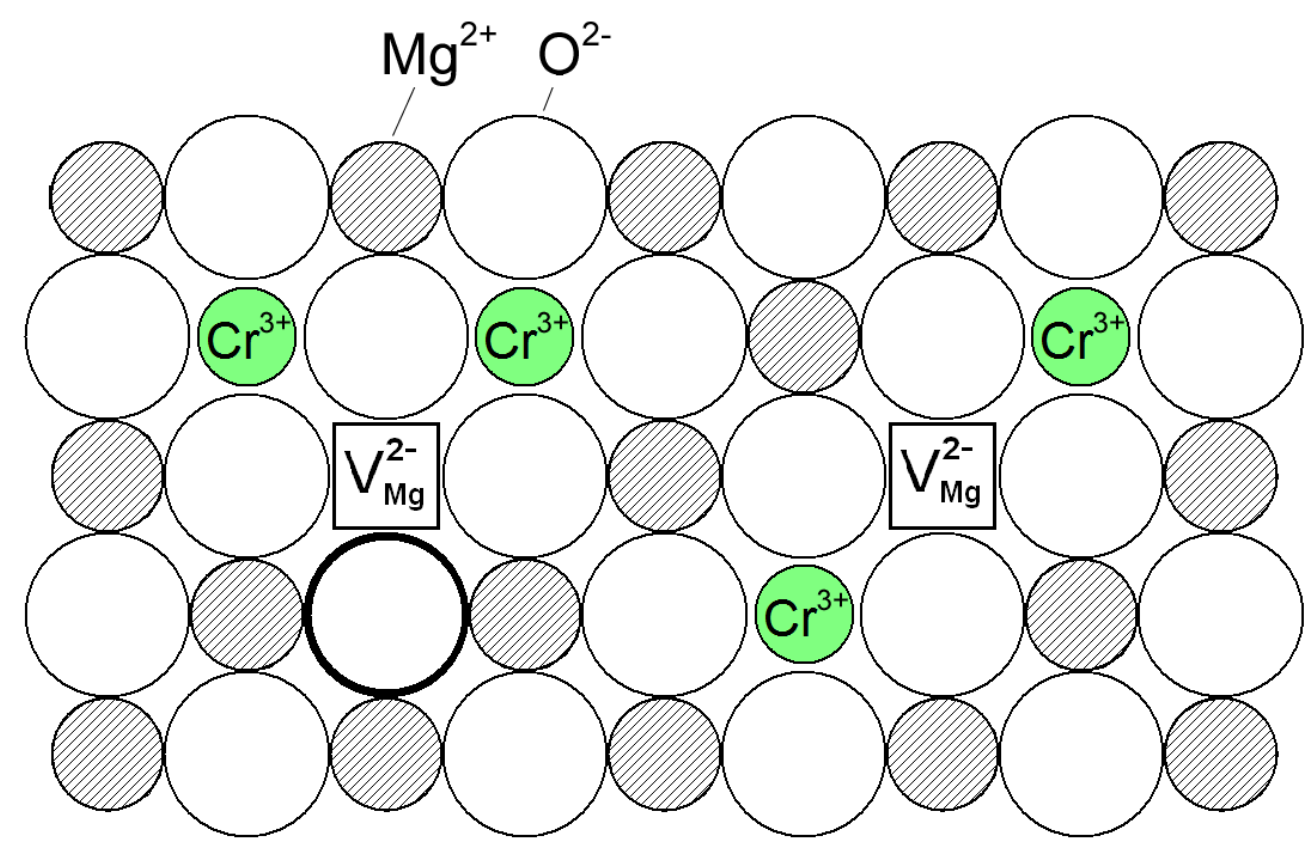

Fig. 1. Possible configurations of two-chromium rhombic centres in $\mathrm{MgO}$. In case of a triangular centre (in the left), a radiation-induced hole becomes trapped at the oxygen ion depicted by a solid circle. 


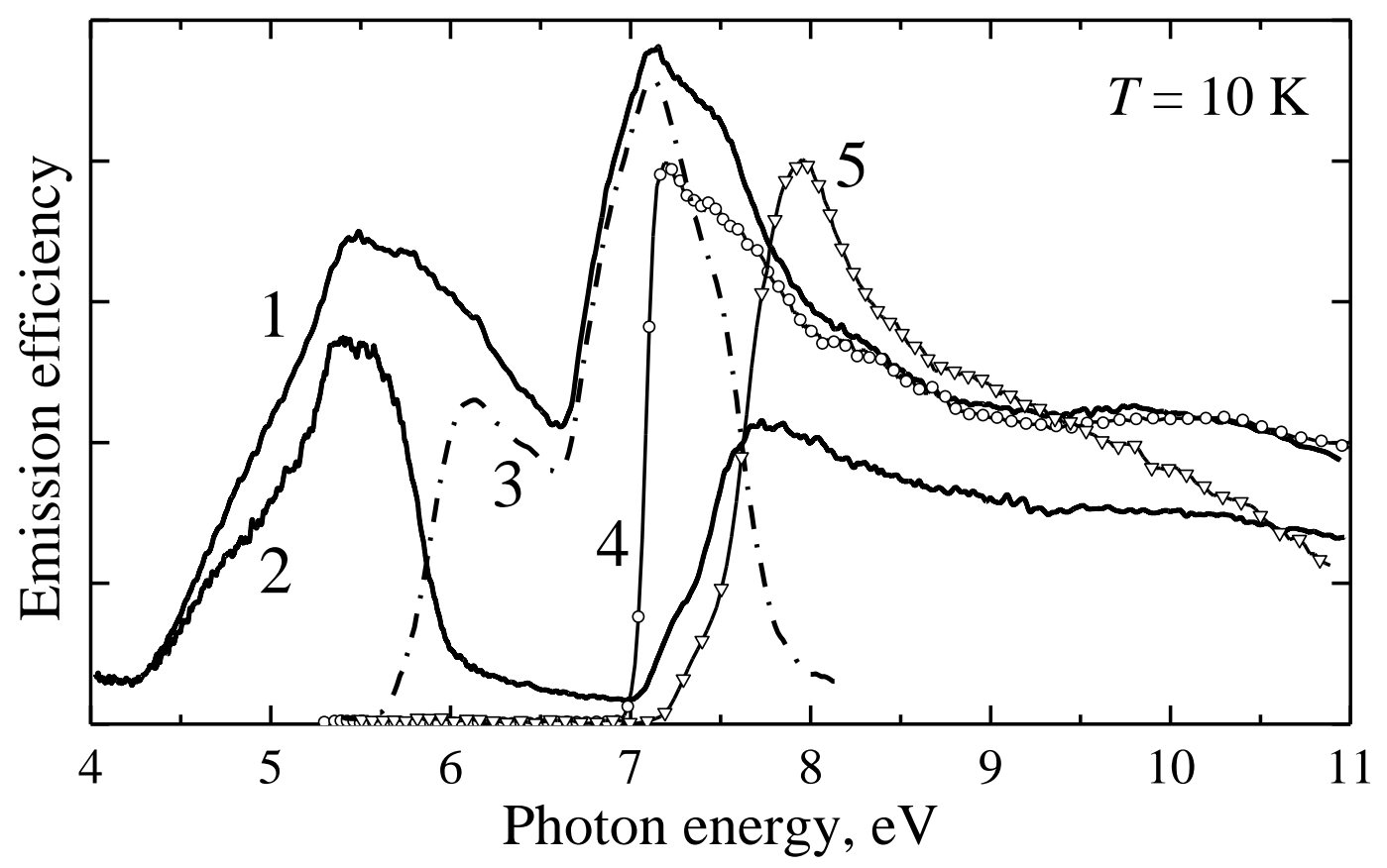

Fig. 2. Excitation spectra measured for the 2.46-eV emission of $\mathrm{Ce}^{3+}$ centres in a virgin (curve 1) and ion-irradiated (curve 2, multiplied by a factor of 8) LuAG:Ce (1400 ppm) single crystals at $10 \mathrm{~K}$. Curve 3 is obtained by a subtraction of curve 2 from curve 1 after their arbitrary normalization at the low-energy maximum. The excitation spectra measured for the 4.5 emission in a virgin (curve 4) and ion-irradiated (curve 5) LuAG single crystals at $10 \mathrm{~K}$. The crystals were irradiated by uranium ions $\left(2.14 \mathrm{GeV}, 10^{12}\right.$ ions $\left.\mathrm{cm}^{-2}\right)$ at $300 \mathrm{~K}$. 


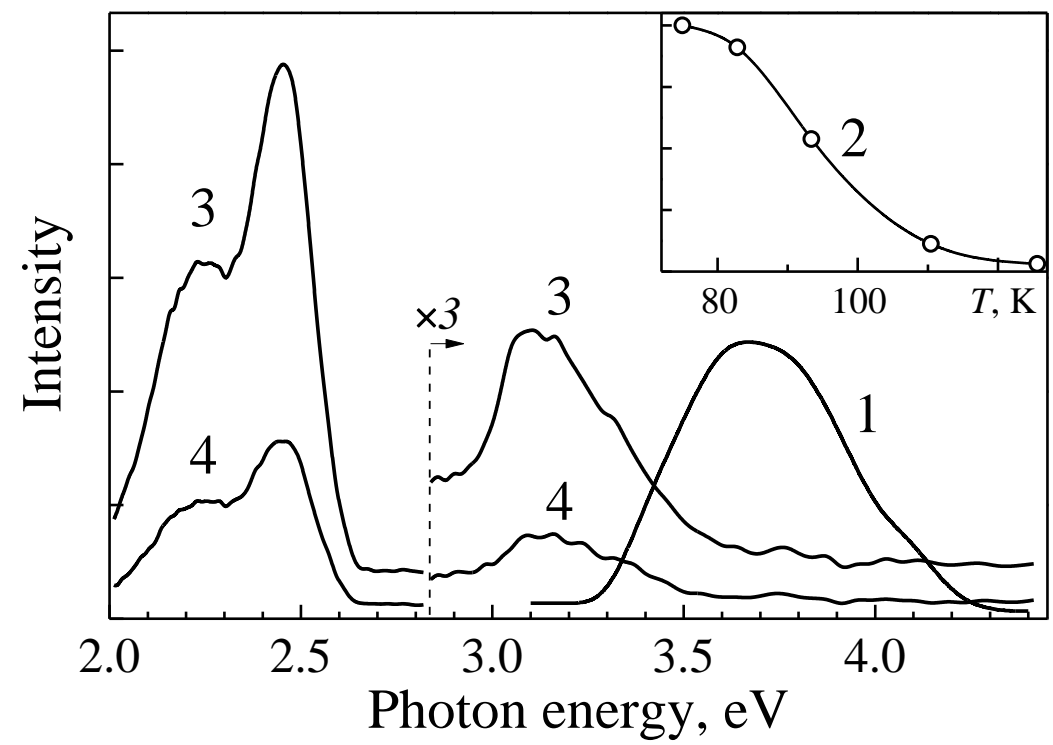

Fig. 3. The enhancement of emission peak amplitude (curve 1) at the excitation by single electron pulses $\left(300 \mathrm{keV}, 3 \mathrm{~ns}, 80 \mathrm{~A} \mathrm{~cm}^{-2}, 80 \mathrm{~K}\right)$ and temperature dependence of the $3.75-\mathrm{eV}$ emission (curve 2) measured for an ion-irradiated $\mathrm{Lu}_{3} \mathrm{Al}_{5} \mathrm{O}_{12}$ crystal $\left(2.14 \mathrm{GeV}, 10^{12}\right.$ ions $\mathrm{cm}^{-2}$, $300 \mathrm{~K}$ ). The emission spectra measured for a time-integrated signal (curve 3) or the emission within a time window ( $\delta t=20 \mathrm{~ns}, \Delta t=30 \mathrm{~ns}$, curve 4$)$ at the excitation of LuAG:Ce ${ }^{3+}(1400$ ppm) with $6.1 \mathrm{eV}$ photons at $10 \mathrm{~K}$. 